with intravenous antibiotic therapy. $81 \%$ of patients had a more than $10 \%$ increase in distance walked with nebulised gentamicin, 61\% with oral therapy and 50\% with intravenous therapy (figure 1).

Conclusions The ISWT is an objective, quick and inexpensive clinical endpoint that is reliable, valid and responsive for use in assessing patients with bronchiectasis.

\section{S46 IS PSEUDOMONAS INFECTION A NECESSARY PRECURSOR TO NTM INFECTION IN NON-CF BRONCHIECTASIS?}

M Elsayed, E Doherty, C Marchand, A Malin. Royal United Hospitals, Bath, UK

\subsection{6/thoraxjnl-2017-210983.52}

Background Non-tuberculous mycobacterial (NTM) infection is more prevalent in those with bronchiectasis than the general population. In addition, Pseudomonas is frequently isolated in more severe bronchiectatic disease. We interrogated our nonCF bronchiectasis database to identify association.

Method A retrospective analysis of 232 patients with non-CF bronchiectasis distinguished those both with and without NTM infection. Analysis included demographic, clinical, microbiologic, lung function and radiological data over a 10 year period.

Results NTM were cultured in 29 patients (12.5\%), M gordonae being the most frequent $(\mathrm{n}=11,37.9 \%)$ followed by $M$ avium-intracellulare $(\mathrm{n}=9,31.0 \%)$. Pseudomonas infection, current or previous, was identified in 146 (62.9\%). Of those with NTM infection, a history of Pseudomonas infection was very strongly associated (96.6\%) with only a single case of NTM isolated without Pseudomonas (3.4\%; $\mathrm{p}=0.001$ ) (figure 1). Also, concurrent proton pump inhibitor use in the NTM group showed a strong association $(55.2 \%$ vs. $29.06 \%$; $\mathrm{p}=0.03)$.

Conclusion A 10 year analysis of our non-CF bronchiectasis cohort indicates a very strong association between prior Pseudomonas infection and subsequent NTM isolation, with an NTM negative predictive value $98.8 \%$ in the absence of Pseudomonas. Whilst association is not causation, we postulate that Psendomonas may lead to specific mucosal microbiome and structural changes. Moreover, this may be a necessary antecedent prior to observing the very high NTM prevalence rates found in this condition.

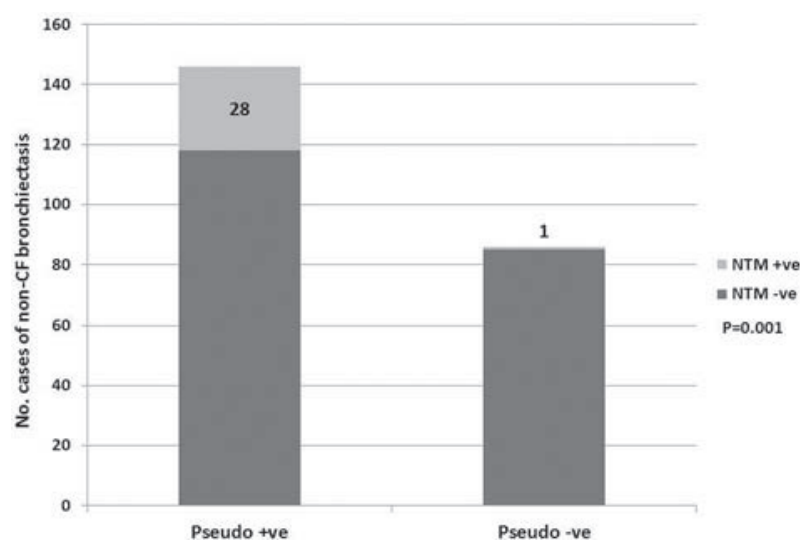

S47 HYPERTONIC SALINE INHALED THERAPY - RESULTS OF DRUG REACTION ASSESSMENTS

${ }^{1} \mathrm{G}$ Rinaldi, ${ }^{2} \mathrm{G}$ Housley, ${ }^{2} \mathrm{~A}$ Shah, ${ }^{1} \mathrm{~B}$ Dennis, ${ }^{2} \mathrm{M}$ Loebinger. 'St.George's University, London, UK; ${ }^{2}$ Royal Brompton Hospital, London, UK

\subsection{6/thoraxjnl-2017-210983.53}

Introduction Hypertonic saline (HTS) is commonly nebulised used to aid airway clearance in patients with chronic suppurative lung disease. In view of the risk of bronchoconstriction, prior to starting HTS, patients undergo a drug reaction assessment (DRA), as suggested by guidelines. ${ }^{1}$ Patients that experience a $>15 \%$ reduction in FEV1 post inhalation, + -lack of tolerability, are deemed to have failed the DRA and would not usually be prescribed it for continued usage. We aimed to identify patient characteristics predicting a successful DRA and the likelihood of continuing HTS at 1 year post DRA.

Methods A retrospective analysis of all HTS DRAs between April 2011 and March 2016 at the Royal Brompton Hospital was undertaken. Spirometry, age, gender and underlying disease were recorded and the variables associated with DRA success and continued use at 1 year were assessed with logistic regression.

Results 523 patients underwent an HTS DRA with overall $89.5 \%$ passing the test. There were 504 tests with $7 \%$ HTS (90.2\% passed) and 18 tests with $3.5 \%$ HTS (73.7\% passed). A higher FEV1\% Predicted Pre-Trial (PPT) was significantly associated with passing the DRA with an Odds Ratio (OR) of 0.97 (95\%CI: 0.95-0.98, p-value <0.001); patients with an FEV $\%$ PPT $>61 \%$ had a $0.05 \%$ chance of failing the DRA. Patients diagnosed with ABPA or COPD were significantly more likely to fail the DRA for HTS with ORs of 3.07 (95\% CI:1.15-8.1, p-value $=0.025)$ and 3.38 (95\%CI:1.06-10.76, pvalue-0.039), respectively. Amongst the 468 patients who passed the DRA, those with a higher FEV1\% PPT were also more likely to remain on the HTS after 12 months, whilst, non-CF Bronchiectasis (OR: 0.44, p-value $=0.020$ ) and patients with "Other" lung diseases, including carcinoma and sarcoidosis, (OR:0.33, p-value $=0.008$ ) patients were significantly less likely to remain on it.

Conclusions The vast majority of patients passed the HTS DRA test and the failure rate in those with FEV1 PPT $>61 \%$ was extremely low. We propose that clinical phenotypes could be used to risk assess patients who need HTS DRA tests before starting HTS.

\section{REFERENCE}

1. Pasteur MC, Bilton D, Hill AT. British Thoracic Society guideline for non-CF bronchiectasis. Thorax 2010;65(1):i1-58. doi:10.1136/thx.2010.136119

\section{Diagnosing and treating pulmonary vascular disease}

\section{S48 SEPTAL ANGLE ON MRI PREDICTS COMBINED PRE AND POST CAPILLARY PULMONARY HYPERTENSION}

${ }^{1} \mathrm{CS}$ Johns, ${ }^{1} \mathrm{JM}$ Wild, ${ }^{1} \mathrm{~S}$ Rajaram, ${ }^{1} \mathrm{E}$ Tubman, ${ }^{1} \mathrm{D}$ Capener, ${ }^{2} \mathrm{C}$ Elliot, ${ }^{2} \mathrm{R}$ Condliffe, ${ }^{2} \mathrm{~A}$ Charalampopoulos, ${ }^{2} \mathrm{DG}$ Kiely, ${ }^{1} \mathrm{AJ}$ Switt. ${ }^{1}$ The University of Sheffield, Sheffield, UK; ${ }^{2}$ Sheffield Pulmonary Vascular Disease Unit, Royal Hallamshire Hospital, UK

10.1136/thoraxjnl-2017-210983.54 
Patients with left heart disease commonly develop pulmonary hypertension (PH), and some subsequently develop pre-capillary vascular remodelling. This combined pre and post capillary pulmonary hypertension (Cpc- $\mathrm{PH})$ is defined as mean pulmonary artery pressure (mPAP) $\geq 25 \mathrm{mmHg}$, pulmonary arterial wedge pressure (PAWP) $>15 \mathrm{mmHg}$ and diastolic pulmonary gradient (DPG) of $\geq 7$. Patients with $\mathrm{Cpc}-\mathrm{PH}$, have a worse outcome and targeted pulmonary vascular therapies may be useful. The aim of this study was to assess MRI measured septal angle in the assessment of $\mathrm{Cpc-PH}$.

Methods Consecutive, incident suspected $\mathrm{PH}$ patients who underwent MRI at a pulmonary hypertension referral centre from April 2012 to October 2015 were assessed. Patients with PAWP $>15 \mathrm{mmHg}$, with right heart catheter and MRI on the same day were included. The diagnostic accuracy of septal angle to identify Cpc-PH was assessed.

Results 2437 patients underwent MRI, 1272 were incident and 227 patients had PAWP >15 mmHg. 163 had MRI and right heart catheter on the same date. The average age was 70 (sd 11), 64\% were female. Systolic interventricular septal angle correlated with DPG $(r=0.735, \mathrm{p}<0.0001)$. ROC analysis showed septal angle was predictive of $\mathrm{Cpc}-\mathrm{PH}$ (defined by DPG $\geq 7)$ with area under the curve $0.90(p<0.0001)$. Analysis of the ROC data showed $160^{\circ}$ septal angle as a threshold predicted a DPG of $\geq 7 \mathrm{mmHg}$, with $74 \%$ sensitivity and $90 \%$ specificity $(\mathrm{p}$-value $<0.0001)$. Systolic interventricular septal angle was predictive of outcome with univariate hazard ratio 1.017 (95\% CI 1.007-1.028, $\mathrm{p}=0.001)$. Dichotomised by median value $\left(149^{\circ}\right)$ the hazard ratio was 3.245 (95\% CI 1.720-6.119, p<0.0001) and log-rank chi-square was 12.387 . Conclusion Interventricular septal angle on MRI can non-invasively predict the presence of an elevated diastolic pressure gradient in patients with left heart disease.

\section{S49 ELICITATION OF DISUTILITY VALUES ASSOCIATED WITH THE MODE OF ADMINISTRATION OF DRUGS ACTING ON THE PROSTACYCLIN PATHWAY IN PULMONARY ARTERIAL HYPERTENSION (PAH)}

${ }^{1}$ EW Davies, ${ }^{2} \mathrm{~S}$ Llewellyn, ${ }^{1} \mathrm{~A}$ Beaudet, ${ }^{2} \mathrm{CE}$ Kosmas, ${ }^{2} \mathrm{H}$ Doll. ${ }^{7}$ Actelion Pharmaceuticals Ltd., Allschwil, Switzerland; ${ }^{2}$ ICON plc, Abingdon, UK

\subsection{6/thoraxjnl-2017-210983.55}

Introduction and Objectives Pulmonary arterial hypertension $(\mathrm{PAH})$ is a rare, incurable disease associated with decreased life expectancy and a marked impact on quality of life (QoL). There are three classes of drugs available for treatment: endothelin receptor antagonists (ERA), drugs acting on the nitric oxide pathway (riociguat and phosphodiesterase type 5 inhibitors (PDE5i)), and drugs acting on the prostacyclin pathway. The latter have widely different modes of administration continuous intravenous infusion, continuous subcutaneous infusion, inhaled and oral - which could be associated with varying treatment burden. Health economic evaluations should incorporate the QoL impact of how drugs are administered. This study aimed to elicit societal utility values associated with different routes of administration of drugs acting on the prostacyclin pathway for use in economic evaluations of $\mathrm{PAH}$ treatments.

Methods A UK general public sample completed the EQ-5D$5 \mathrm{~L}$ and valued four health states in Time-Trade Off interviews. The health states (drafted from literature and interviews with
PAH experts $(n=3)$ contained identical descriptions of PAH and ERA/PDE5i treatment, but differed in description of prostacyclin treatment administration including: oral (tablets), inhaled (nebulizer), continuous subcutaneous infusion, and continuous intravenous infusion.

Results A total of 150 participants (63\% female; mean age 37 years) completed interviews. Utilities are presented as values between 1 and 0 , where 1 is equal being in a state of 'full health' and 0 is equal to being dead. The mean (SD) utility for the oral health state was $0.84(0.16)$, while the other health states were all significantly lower at $0.73(0.27)$ for inhaled $(p=0.001), 0.58(0.31)$ for subcutaneous $(p<0.001)$, and $0.54(0.32)$ for intravenous $(\mathrm{p}<0.001)$. Utility differences compared to the oral health state showed that there are disutilities (negative differences) associated with the inhaled, subcutaneous, and intravenous continuous modes of treatment administration. Disutilities were -0.11 for inhaled, -0.26 for subcutaneous, and -0.30 for intravenous administration.

Conclusion The Results demonstrate quantifiable QoL differences between modes of administration of drugs acting on the prostacyclin pathway, so as to allow appropriate reflection of the unique QoL burden within an economic evaluation of drugs for PAH treatment.

\section{S50 CAMPHOR SCORE: SUSTAINED IMPROVEMENT IN PATIENT REPORTED OUTCOMES FOLLOWING PULMONARY ENDARTERECTOMY IN CHRONIC THROMBOEMBOLIC PULMONARY HYPERTENSION}

${ }^{1} \mathrm{M}$ Newnham, ${ }^{1} \mathrm{~K}$ Bunclark, ${ }^{1} \mathrm{~N}$ Abraham, ' $\mathrm{L}$ Amaral Almeida, ${ }^{1} \mathrm{~J}$ Cannon, ${ }^{1} \mathrm{~S}$ Clare, ${ }^{1} \mathrm{~N}$ Doughty, ${ }^{1} \mathrm{~J}$ Dunning, ${ }^{1} \mathrm{C} \mathrm{Ng},{ }^{1} \mathrm{~A}$ Ponnaberanam, ${ }^{2} \mathrm{~S}$ Scholtes, ${ }^{1} \mathrm{~K}$ Sheares, ${ }^{1} \mathrm{~N}$ Speed, 'D Taboada, 'M Toshner, 'S Tsui, 'D Jenkins, 'J Pepke-Zaba. 'Papworth Hospital, Cambridge, UK; ${ }^{2}$ University of Cambridge, Cambridge, UK

\subsection{6/thoraxjnl-2017-210983.56}

Introduction The CAMPHOR (Cambridge Pulmonary Hypertension Outcome Review) score is an internationally validated patient reported outcome (PRO) measure for pulmonary hypertension, including chronic thromboembolic pulmonary hypertension (CTEPH). It assesses 3 areas: activity (score 0 30 ), symptoms (0-25) and quality of life (QoL) (0-25); with a higher score indicating a worse PRO. CTEPH frequently causes debilitating symptoms and functional impairment, which can be improved in selected patients with pulmonary endarterectomy (PEA). However, a subset will have residual pulmonary hypertension. We aim to assess PROs in patients with CTEPH undergoing PEA.

Methods Consecutive CTEPH patients undergoing PEA from June 2006 to August 2016 at the UK National PEA centre, were included in this retrospective analysis. Patients are reviewed after PEA every 6-12 months for at least 5 years. CAMPHOR scores were recorded prospectively when patients attended hospital assessment and at each follow-up, ensuring high capture.

Results 1151 patients underwent PEA during the study period. Of those, $937(81 \%)$ had a CAMPHOR score recorded at baseline (pre-PEA) and 816 (77\% of 1059 alive) at follow-up within a year of PEA (post-PEA). We confirmed significant improvements in $6 \mathrm{~min}$ walk distance and haemodynamics post-PEA (pre/post-PEA median \pm IQR: 6 mwd 300 199 Metres/360 $\pm 165 ; \quad$ mPAP $45 \pm 15 / 25 \pm 13 \mathrm{mmHg} ; \quad$ PVR 669 $\pm 478 / 246 \pm 214$ dynes.s.cm ${ }^{-5} ;$ CI $2.2 \pm 0.8 / 2.3 \pm 0.7 \mathrm{~L} / \mathrm{min} / \mathrm{m}^{2}$ ). The difference in median CAMPHOR scores pre- and post- 